\title{
Multi-Source Data Accordance and Database Construction for City Planning
}

\author{
Xia Xiaotang \\ Wuhan University of Science and Technology \\ College of Urban Construction \\ Wuhan, China \\ xiaxiaotang2010@163.com
}

\begin{abstract}
Urban planning information assembles multiple source data. According to different criterion, urban planning data have different classification. With the computer technology, 3S, OA and workflow, urban planning multiple source date accordance on different coordinate system, data format, data structure, scale and tense aspect is analyzed. Combined with GIS database design trait, based on urban planning database function frame, urban planning spatial database and attribute database structure design are discussed; urban planning database accordance flow is summarized.
\end{abstract}

Keywords—urban planning; GIS; data accordance; database

\section{INTRODUCTION}

Urban planning and management is an accumulatingdisseminating process of a lot of spatial information. With the acceleration of urbanization, the spatial information serving the planning and management has been quickly developed, and the basic geographic data, planning approval data and socioeconomic data have formed the huge information flow. The information is various in different aspects, levels and types, and there are information supporting urban planning or produced by planning, covering and the science and specialty. These multi-type, multi-temporal and multi-resolution graphics, images, text and other heterogeneous data are organically integrated to construct the urban planning database, forming the information resources that can provide urban planning with the qualitative, quantitative, positioning analysis and aid decision-making, which can record and reflect the urban history, status quo and planning, and easily and promptly update the data to achieve mass storage, efficient management, continuous updating and good service, provide important database resources for the construction of "digital city" and provide detailed geographical criteria for the society, government and relevant departments. Urban general planning and the application of urban regional transportation planning subsystem $^{[1]}$.

First, the era of big data on the continuous development of urban planning. Before the arrival of the era of big data, our urban planning is traditional, artificial, with great uncertainty. Analysis of urban space is carried out manually, and the data is not objective. The data used in urban planning is usually obtained through questionnaires and field trip. The arrival of the era of big data, brings new blood to urban planning, but also brings new opportunities. The first change is that big data provides strong data support for urban planning, laying the foundation for the scientific nature of urban planning. In the era of big data, city planners will have clearer knowledge and understanding of the city. They can use the positioning system to understand the distribution of the city space, and to make scientific planning, such as population density, city living Nebula map balance, city traffic distribution, and population flow of scientific analysis. Secondly, another feature of the era of big data is "big", it has a large capacity of city road traffic conditions and distribution characteristics of various regions and various geographic information features accurate and detailed access. Therefore, it is more convenient and scientific for urban planners to analyze and study urban space. Big data for the city's construction planning to provide a professional data support, so that it can make urban planning more scientific

Application of development of big data in urban planning. Big data under the urban planning mainly rely on big data on the distribution of urban space in China to obtain a reasonable scientific planning of urban space. City is the living environment for people; people are the leaders of citys. Therefore, some people's daily activities will affect the distribution of urban space in China. The analysis results of big data can accurately analyze people travelling, shopping, entertainment, life characteristic ${ }^{[2]}$. The analysis results can support the regional center of the city planning and design of the layout of public facilities and traffic planning and configuration, and can also be more scientific to effectively solve the evening rush hour congestion and unreasonable public facilities layout problems. Big data is not only effective for the relevant staff in the process of urban spatial planning and to provide a more intuitive understanding, but also to ensure the greatest degree of reasonable distribution of urban space in China.

Second, the advantages of big data in urban planning. In line with the trend of development of contemporary urban planning. With the development of the times, the traditional urban planning has not been able to keep up with the trend of the development of our times. The traditional urban planning has a certain lag to the city planning. The means of data access by traditional city planning is single and simple, or even backward, which has leaded certain one-sided problems on analysis and processing of various data. The city planning not only plays a positive role, but caused adverse effects. The traditional urban planning is not only adapting to the development of China's urban economy, but also hinders the 
development of China's urban economy. Big data is different, it makes the city planning more scientific, which can effectively promote the development of urban economy. Big data can improve the efficiency of urban planning, reducing the waste of resources to save costs ${ }^{[3]}$. Traditional urban planning in the analysis of statistical data and data is usually done by people. This not only increases the workload of the staff, but also cannot guarantee the accuracy of the relevant data. This is not conducive to the city's scientific and rational planning. In the era of big data, city planning is related to the data collected by information technology, based on the city planners in the city space for further study, and then to the city to carry out scientific and reasonable planning. This not only makes China's urban space has been a reasonable scientific planning, but also reduces the waste of human resources, truly achieve the best".

\section{Multi-SOURCE DATA ANALYSiS Of URBAN PlANNING}

In the urban planning and management information system, the data sources are different in different forms, and their functions are also different. In order to integrate these multisource data organically, it is needed to correctly understand and classify the multi-source data, so as to use them reasonably in building and implementing the system ${ }^{[4]}$.

Urban planning data can be divided into planning data and basic data according to the content, the former of which includes the planning maps, corresponding technical indicators and relevant planning parameters. According to the data use of planning and management department and the data processing methods, the planning data are also subdivided into five types: land balance, planning scope, architectural form, planning parameter and schematic element. The basic data mainly include the urban status, traffic and socioeconomic data.

The urban planning data can be divided into the following parts according to the data composition: 1) Basic map data, including topographic maps and remote sensing images; 2) Resource and environmental data, including geology, hydrology, water conservancy, vegetation, soil, minerals, cultural relics and environmental protection; 3) Social and economic data, including the socio-economic information provided by government statistical departments and collected by planning departments, also including the relevant laws and standards; 4) Land use data, including the status quo, historical conditions, permission for land use and construction, also including the parcel boundary and ownership in land management and in real estate management; 5) Municipal and public facilities data, mainly including various municipalities, transport, public utilities facilities, public services, social welfare, and disaster relief facilities; 6) Planning result data; 7) Management business file data; 8) Daily management case data, mainly including all kinds of planning and management.

\section{URBAn PLANNING MULti-SOURCE DATA INTEGRATION}

\section{A. Urban planning multi-source data integration content}

1) Integration of multi-source data space coordinate system

Spatial data is an important part of urban planning data. During the urban planning data, the spatial data are from different departments or times, so it is inevitable that these spatial data have different coordinate systems, so the spatial data of different coordinate systems must be integrated into the unified coordinate system, so as to facilitate the implementation of data analysis.

\section{2) Integration of spatial data and attribute data}

At the beginning of urban planning and management information system construction, many spatial data are managed via AutoCAD, and forms are managed via Excel, but these two-relevant data are stored independently, easily causing the inconsistency of data update. To build the urban planning database, it is needed to integrate spatial data and its corresponding attribute data, establish the association between spatial data and attribute data, and realize the integrated storage and management of spatial data and attribute data, so as to provide technical support for achieving distributed information processing and spatial data sharing.

\section{3) Integration of data in different formats}

During the construction of urban planning database, the data are transferred from the original source to each department and further to the urban planning and management information system. To meet different needs for each department, a variety of professional geographic information system and cartographic software are needed in each step in the data acquisition and processing; besides, different professional geographic information system software has its own relatively independent data format, so it is needed to convert different formats of data into the unified data format during the construction of urban planning database.

\section{4) Integration of data in different scales}

In the urban planning and management information system designed based on GIS, different scales can be set for different spatial data according to the requirements or sources, and it is not necessary to set the same scale for all graphics; but as for the different spatial data under the same scale, different scales can be set according to the different content, and the system will determine which spatial data are to be displayed according to the current view scale, so as to achieve the non-level display and automatic switching of spatial data ${ }^{[5]}$.

\section{5) Integration of vector data and raster data}

Vector data and raster data are two kinds of the most important data models in GIS system. These two data models are complementary to each other in advantages and disadvantages. Only by integrating the vector data accurately described by coordinates and the image data described by the raster organically can they complement each other in the data analysis, providing more accurate and clear information for the planning design and planning management and improving the scientific nature of urban decision-making.

6) Integration of history, status quo and planning data

Urban development has obvious temporal characteristics, so urban planning and management information system should also be able to reflect the development situation in different times, which is not only the urban planning for future development, but also the scientific management and reproduction of urban history and status quo data. Only by correctly analyzing the urban history and status quo data can 
we make scientific and rational planning for future urban development. Therefore, it is needed to organically integrate the urban history, status quo and planning data.

\section{B. Key technology in urban planning multi-source data integration}

The construction of urban planning and management information system is closely related to the development of information technology with different development tracks in different historical periods. With the development of computer hardware and software technology, database technology and GIS, etc., the construction of urban planning and management information system and multi-source data integration are inseparable from the following key technologies.

\section{1) Computer and network technology}

In order to maximize the advantages of computer and network, the urban planning and management information system should make full use of the computer and network technology to acquire, process, store, supervise and manage the data, so as to realize the integration of urban planning approval, planning design and planning management, and realize the "paperless office".

\section{2) $3 S$ technology}

The application and development of geographic information system, remote sensing and global positioning system provide a more convenient pathway for the information storage, management, analysis and updating. The urban planning and management information system should fully integrate 3S technology to integrate and operate its multi-source data.

\section{3) Office automation (OA)}

Administrative office is an important content of urban planning and management information system. In order to fully realize the "paperless" and "automation" office and simplify the complex office program, the system must be connected with OA seamlessly, realizing the interoperability of graphics, attributes and document data and construction of spatial data and attribute data.

\section{4) Workflow technology}

The urban planning and management is a very work with high workflow, so the multi-sectoral coordination is needed. The internal workflow, operation approval, automatic information transmission in office, information exchange, approval process tracking and progress control need the support of workflow technology. The introduction of workflow technology can realize the seamless integration of urban planning and management business office automation, business processing and window, and fuse the office system and GIS system.

\section{URBAN PlanNing DAtabase CONSTRUCTION}

In the construction of urban planning and management information system, the database construction is the core content, the reliability of the data, the rationality of the data organization will directly affect the success or failure of the system database, but also the key to the whole system. Scale, multi-level and other characteristics determine the complexity of planning data, only the effective organization and management of these data, so that they can constitute a whole to complement each other and reference in order to give full play to the role of data for easy inquiry, analysis and decisionmaking.

\section{A. Database construction standards}

In order to realize the seamless connection and integration of multi-source data and ensure the consistency, compatibility, convertibility and integrity of urban planning information, it is necessary to formulate a standard system. When you develop, you should determine the required standard category, To understand the specific content of each type of standards, with local, national and international standards, to identify their local standards, national standards and international standards of the status quo and development trends; down to highlight their own characteristics, give full consideration to urban planning Special requirements to meet the application of urban planning and management needs and development needs; some aspects of the lack of standards to follow the local industry norms and rules in short, the development of the standard system to fit the specific circumstances of urban planning is conducive to the promotion of urban planning Information production, updating, management, distribution and application services.

(1) To establish a unified urban planning spatial positioning benchmarks, including a unified urban coordinate system, map sub-numbering rules to determine the basic map of the basic map of the city;

(2) To follow a unified urban measurement and engineering measurement specifications, including urban basic map mapping and mapping, urban infrastructure mapping and mapping, construction project acceptance measurement and mapping, urban infrastructure measurement and mapping and other norms and standards;

(3) To establish a unified data standard, including data classification, hierarchical and coding rules, planning and construction review data standards, the preparation of the results of data standards, data dictionary and the database file naming standards, data formats and exchange standards;

(4) Standardize the operation and operation procedures, including graphic information and database operation procedures, data quality control standards, database maintenance processes, various forms, text and other document information format standards;

(5) Metadata standards that embody the characteristics of urban planning information;

(6) To establish a relatively sound and reasonable data dynamic update mechanism to ensure data consistency, integrity and current potential.

\section{B. Database Functional Framework}

1) Unified data management

The establishment of a unified document and spatial database management system, so that a variety of data can be quickly and easily in the planning and management call 
superposition, while effectively improving the efficiency of the system and to ensure data reliability and security.

2) Efficient data exchange and data sharing

The relevant departments and planning branch to achieve efficient data exchange, so that between the various departments to achieve real data sharing.

\section{3) Data storage and backup}

Through the appropriate storage and backup strategy for the data in the database for secure storage and backup to ensure data security, to ensure uniform and smooth.

4) Heterogeneous database interconnection

Different database platforms, different network transmission platform data security connection and summary.

\section{Database structure design}

1) Spatial database structure design

The spatial data used in the urban planning and management information system is a kind of vector structure data expressed by points, lines and faces, and the lattice structure data such as remote sensing images and pictures expressed by grid cells are used. Due to the large amount of land Using the current situation data, the planning land data is basically in the form of vector $^{[5]}$. This part discusses the spatial database structure design based on the vector data model.

Urban planning and management information system is a kind of practical GIS, and GIS system generally according to the needs of system data organization, the use of graphical hierarchical technology to organize vector data, that is, composite graphics data into different layers. In the system database construction, the first Should be different types (points, lines, faces) of the graphical object storage; for the basis of the information can be derived from the geographical data, should also be stored in a separate layer, such as urban land use status data can be derived from different types of land or different partitions Of the land use data, the data can not be mixed with other surface data in a layer; In addition, for different application purposes and the use of the frequency of data should also be based on different data storage methods to separate the city land use planning data as an example.

\section{2) Attribute database structure design}

After the integration of urban planning spatial data and attribute data has become inseparable unity, the attribute data structure is determined by the characteristics of the spatial data associated with it, but also by the functional objectives of the system, such as planning data can contain Such as land use type, land area, building control volume rate, building control height and so on.

\section{3) Meta-database design}

There are a lot of spatial graphic data and related attribute data or pictures, images, sound and image data in the city planning database, which must be described and explained in the understanding, utilization or query, statistics, analysis of these data. In addition to the content of the data dictionary in addition to the contents of the data dictionary should be included in the database of some of the data on the unique information on the vector-type planning data, the metadata generally include: (1) data topic description, including the subject name, release date;(2) the description of the data structure of the data; (3) the description of the data, including the data source, the production date, the projection, the coordinate system and so on; (5) the description of the storage path and the structure. Especially the remote sensing image data, the metadata should describe the type of image, coverage, size, intake time, projection center position and so on.

\section{SUMMARY}

The development of geographic information system and database technology provides a broad space for the management and performance of multi-source data.

The GIS software platform and the database management software are used to realize the unified storage of multi-source data in the city. However, it is still necessary to use many standards in the integration, integration, management, sharing and data mining of urban heterogeneous data. The establishment of the support of the city planning and multisource data integration and database construction is a long and arduous task.

\section{ACKNOWLEDGMENT}

The research is supported by the humanities and social science research project of Hubei Provincial Education Department (NO: 15Q032)and supported by the scientific research project of Hubei Provincial Education Department (NO: Q20141106)

\section{REFERENCES}

[1] Hu Ling, Liu Qiang.Application of ArcSDE Geodatabase to GIS Application of Urban Planning Management [J] .Computer Science, 2006, 33 (12): 125-127.

[2] Sui Mingming, Li Zonghua.Study on the realization of spatial data sharing and interoperability in planning land management [J]. Geospatial Information, 2005, 3 (3): 16-18.

[3] Lu Xinhai.Multi-source urban planning data fusion and integration method [J] Surveying and Spatial Geographic Information, 2005, 28 (4): 47-49.

[4] Li Heng, Yang Dan, Fang Weitao, et al. Design and research of workflow management system [J] .Computer Science, 2008, 35, (8): 293-296.

[5] Zhou Mei, Yang Kai, Tan Shunping.Application of ArcSDE and Oracle Spatial Data Integration [J]. Journal of Guangxi University: Natural Science Edition, 2007, 32 (4): 407-410. (Responsible Editor Liang Bifen) 110 Guangxi Journal of the University of Science and Technology. 